\title{
Editorial
}

\section{Special Issue on Dynamic Analysis and Testing of Embedded Software}

Research challenges in dynamic analysis and testing have been studied for decades. Nonetheless, as new hardware and software technologies emerge and become more powerful, many interesting techniques that were previously considered to be impractical or remained unexplored are receiving significant attention. This special issue presents novel research work in the dynamic analysis and testing of embedded software, which also comes with theoretical or extensive empirical validation of the claimed contributions. Out of 15 submissions, four are accepted for publication based on the reviewers' professional assessments and recommendations.

The first paper, "InRob: An Approach for Testing Interoperability and Robustness of Real-Time Embedded Software" by F. Mattiello-Francisco, E. Martins, A.R. Cavalli, and E.T. Yano, presents an approach known as InRob to support model-based integration testing of components with timing constraints. The work focuses on the aspects of interoperability and robustness. InRob uses a state-based model to represent each component, and applies the notion of timing hazard as abnormal timing deviation, which ultimately leads the abovementioned state-based models to specify new faulty states or transitions with timing requirements. The approach then generates model-based test cases to cover these states and transitions when these state-based models are composed together. A case study on the software embedded in a real-time space X-ray telescope is presented to evaluate the feasibility of the approach in practice. The result of the case study shows that the approach reveals a rushed message hazard that has not been reported previously.

The second paper, "Test Coverage Optimization for Large Code Problems" by Y.-D. Lin, C.-H. Chou, Y.-C. Lai, T.-Y Huang, S. Chung, J.-T. Hung, and F. C. Lin, focuses on the regression testing of software with large codebase. They study the tradeoff between minimizing the total regression time cost and minimizing the total number of test cases in a reduced test suite via six existing strategies, which are implemented as six techniques. They use the MPLS code in the Cisco Internetwork Operating System as the subject in an empirical study. The result shows that the removal of infrastructure modules from the consideration of test case selection only trades the fault detection ability and code coverage on other components by a few per cent, and out of the six techniques, PDF-SA can significantly reduce the time cost incurred by other techniques studied. Furthermore, the empirical result also shows that the selection of an appropriate effective-confidence level is important in striking a balance between the factors of cost and coverage.

In performance testing, a technique incurring a high run-time overhead is undesirable because it adversely affects the software under study. The third paper, "Light- weight Embedded Software Performance Analysis Method by Kernel Hack and its Industrial Field Study" by J. Seo, B. Choi, and S.-W. Yang, presents a method for the performance testing of embedded software, which aims at minimizing the perturbation of the software environment with low run-time overhead. The authors insightfully identify that a process control block in an operating system contains all the execution information related to a process, which manifests as a process running on top of the OS and can be used to spot performance bottlenecks in an application. Their technique probes such execution information for the software under test. The authors use a case study of the embedded software in a car navigation system to validate the run-time overhead and the accuracy of their technique. The case study shows that the technique can help locate system performance bottlenecks with 6 Kbytes of code-expansion factors and $11.93 \%$ of slow-down factors.

The fourth paper, "Automatic Testing Environment for Multi-Core Embedded Software - ATEMS" by C.-S. Koong, C. Shih, P.-A. Hsiung, H.-J. Lai, C.-H. Chang, W.C. Chu, N.-L. Hsueh, and C.-T. Yang, identifies six issues in the testing of embedded software in multi-core platforms. To automate some activities, a testing environment, ATEMES, has been created with more advanced features than existing tools. To address the resource constraint problem when testing software on a target platform, the technique proposes multi-round performance testing using the Intel TBB library to systematically locate desirable values for parameter token numbers to improve the system-wide parallelism. Other automation includes remote code compilation and deployment as well as test coverage and CPU core monitoring to track the concurrency and performance issues. The work also presents a usability evaluation by using more than 30 students as human subjects and software running on an ARM11 multi-core platform as the program subject. The empirical findings show that the use of the tool can significantly help human subjects complete the tasks efficiently for the testing of embedded software.

We are grateful to the authors for sharing their research ideas and results with readers and to the reviewers for their valuable feedback on the papers. Special thanks also go to the Editor-in-Chief, Professor Hans van Vliet, for his continuous support and encouragement. We would also like to thank the Journal of Systems and Software for hosting this special issue.

W. Eric Wong received his Ph.D. in Computer Science from Purdue University. He is currently an Associate Professor and Director of International Outreach in Computer Science at the University of Texas, Dallas. Prior to joining UTD, he was with Telcordia (formerly Bellcore) as a Project Manager for Depend- 
able Telecom Software Development. Dr. Wong received the Quality Assurance Special Achievement Award from Johnson Space Center, NASA, in 1997. His research focus is on the technology to help practitioners develop high quality software at low cost. In particular, he is doing research in software testing, debugging, safety, and reliability. Dr. Wong is Vice President-elect for Technical Operations of the IEEE Reliability Society, and the Secretary of the ACM Special Interest Group on Applied Computing (SIGAPP).

W.K. Chan is an assistant professor at City University of Hong Kong. He received all his degrees from The University of Hong Kong. He worked in the industry for many years before returning to academia to complete his $\mathrm{PhD}$ degree and starting his academic career. He is on the editorial board of Journal of Systems and Software. He is serving or served as guest editors of international journals and as program or track chairs of APSEC 2012, QSIC 2010, and AST 2010. His latest primary research interest is to address the software analysis, testing, and debugging issues in assuring large-scale software applications. His work has been reported in many international conferences and journals such as TOSEM, TSE, CACM, ICSE, FSE, ICDCS, WWW, and ASE.

T.H. Tse is a professor in computer science at The University of Hong Kong. He received his $\mathrm{PhD}$ from the London School of Economics and was twice a visiting fellow at the University of Oxford. His current research interest is in program testing, debugging, and analysis. He is the steering committee chair of QSIC and an editorial board member of the Journal of Systems and Software; Software Testing, Verification and Reliability; Software: Practice and Experience; and Journal of Universal Computer Science. He is a fellow of the British Computer Society, a fellow of the Institute for the Management of Information Systems, a fellow of the Institute of Mathematics and its Applications, and a fellow of the Hong Kong Institution of Engineers. He was decorated with an MBE by The Queen of the United Kingdom.

Fei-Ching Kuo is currently a senior lecturer in Swinburne University of Technology. Her research interests include software testing, debugging, and project management. She has been an IEEE member for many years, a co-editor of special issues for Journal of Systems and Software, Software: Practice and Experience, and International Journal of Software Engineering and Knowledge Engineering, a program co-chair for QSIC 2010, a PC member of international conferences and workshops, including ISSRE, COMPSAC, and SAC amongst others, and also acted as a reviewer for several international journals and conferences.
Guest Editors

W. Eric Wong*

The University of Texas at Dallas, Richardson, TX, USA

W.K. Chan

City University of Hong Kong, Tat Chee Avenue,

Hong Kong

T.H. Tse

The University of Hong Kong, Pokfulam, Hong Kong

Fei-Ching Kuo

Swinburne University of Technology, Melbourne, Australia

* Corresponding author.

E-mail addresses: ewong@utdallas.edu (W.E. Wong), wkchan@cs.cityu.edu.hk (W.K. Chan), thtse@cs.hku.hk (T.H. Tse), dkuo@swin.edu.au (F.-C. Kuo) 